\title{
Autowaves in the Model of Infiltrative Tumour Growth with Migration-Proliferation Dichotomy
}

\author{
A.V. Kolobov ${ }^{1}$, V.V. Gubernov ${ }^{1}$ and A.A. Polezhaev ${ }^{1}$ * \\ ${ }^{1}$ Department of Theoretical Physics, P.N. Lebedev Physical Institute, \\ Leninskij prosp. 53, Moscow 119991, Russia
}

\begin{abstract}
A mathematical model of infiltrative tumour growth is investigated taking into account transitions between two possible states of malignant cells: proliferation and migration. These transitions are considered to depend on oxygen level in a threshold manner where high oxygen concentration allows cell proliferation, while concentration below a certain critical value induces cell migration. The infiltrative tumour spreading rate dependence on model parameters is obtained. It is shown that the tumour growth rate depends on the tissue oxygen level in a threshold manner.
\end{abstract}

Key words: tumor growth, proliferation and migration dichotomy, oxygen AMS subject classification: 92B05, 35K40

\section{Introduction}

Malignant tumours are one of the main causes of mortality in developed countries, so cancer research and treatment has become increasingly important in the modern society. Significant efforts are made to cope with cancer but the problem is still unsolved. There is a large variability in malignant neoplasms properties as there are more than 100 types of cancer, each having a lot of subtypes. However, it has been hypothesized [10] that nearly all cancers develop a common set of basic characteristics: (1) self-sufficiency in growth signals, (2) insensitivity to anti-growth signals, (3) evasion of apoptosis, (4) limitless replicative potential, (5) sustained angiogenesis and (6) tissue invasion and metastasis. By focusing on these common elements, mathematical modelling aims to contribute to the prevention, diagnosis and treatment of this complex disease.

*Corresponding author. E-mail: apol@1pi.ru 
From mathematical point of view, one of the cancer hallmarks, an unlimited proliferation, implies a malignant cell number exponential growth with time. In vivo experiments and in vitro study of multicellular tumour spheroids have demonstrated that the initial exponential phase of growth is followed by the logistic phase, which can be described by the Gompertz equation [15]. This transition occurs due to nutrient depletion in the tumour interior that stops proliferation and causes necrosis. Thus, necrotic area can be found in the tumour center while dividing cells are situated in tumour boundary. Tumours with such a structure are referred to be in a diffusion limited phase of growth. In 1955 Thomlinson and Gray [21] proposed a mathematical model for oxygen diffusion and consumption in order to support experimental investigation of some bronchial carcinomata types. In 1966 Burton [1] developed a diffusion model, which described both the distribution of oxygen in a spherical tumour and the radius of the central necrotic area. It was demonstrated that the tumour growth curve obtained in the model could fit a Gompertzian expression. Later many papers were published where the tumour cell mitotic index (proliferation rate) was found to directly depend on nutrient (usually oxygen or glucose) concentration [2, 3, 8].

Malignant cell migration is another critical property which defines tumour growth and progression. There are many types of tumours where malignant cells are considered to be practically immotile. Tumour grows like a compact object with the total malignant cell density close to the maximum permissible cell density in tissue. Local changes in cell population due to a division or a cell loss result in the internal pressure variations, which in turn induces migration and tumour enlargement. Convection-dominated mathematical models, where tissue with a tumour is considered as incompressible fluid and cell motion is determined by convective field, are used to describe this type of cancers $[20,22]$. In models of such type there are several convection equations describing dynamics of different tumour cell types or phases and one or more reaction-diffusion equations for nutrients and/or drugs.

Tumours with high individual motility of their cells are also known. Glioma is a striking example of such tumours called infiltrative [4]. Tumours of this type are characterized by a rather low fraction of malignant cells in the tissue and an extensive penetration into the normal surroundings provided by high individual cell motility. A general mathematical description taking into account both convective fluxes and individual cell motility was developed by Gusev and Polezhaev [9]. Such type of models [14] is rather resource-consuming for numerical solution even in 2-D. Usually for infiltrative tumours with low relative cell density the convective fluxes may be neglected and thus the model can be reduced to several simple reaction-diffusion equations for cell densities, nutrients and/or mediator concentrations $[18,19]$. These models are able to describe linear dependence of tumour size on time obtained in experiments. It was shown that the diffusion approximation for the transport process together with a logistic growth yields an overestimation of the overall growth rate [5]. Moreover, there are experimental data obtained in vitro and clinical data obtained in vivo which indicate an inverse correlation between motility and proliferation of tumour cell phenotypes [7]. It was called "a migration-proliferation dichotomy". Tumour cells proliferation and migration turned out to be mutually exclusive phenotypes: the spreading suppresses cell proliferation and vice versa. In 2006 Iomin [13] formulated migration-proliferation dichotomy in the framework of the continuous time random walk transport concept. This approach based on jump and waiting-time distributions has been used for modelling of glioma cell invasion [6]. In 
that paper only stochastic switching between proliferation and migration was considered.

Another type of mathematical models useful for migration-proliferation dichotomy investigation is discrete models. As they are based on a series of rules for each cell, it is possible to translate detailed biological processes (e.g. cell-cycle events, migration, mutation pathways) into simple rules for the model [16]. This approach is particularly useful for studying carcinogenesis, natural selection, genetic instability and interactions of individual cells with each other. On the other hand, the computational cost increases rapidly with the number of cells modelled, limiting these methods in the spatial and temporal scales they can achieve. In 2010 Hatzikirou and co-authors [12] used a lattice-gas cellular automaton model for investigation of migration-proliferation dichotomy as a potential mechanism that may promote the progression from benign neoplasms to malignant invasive tumours characterized by high migration rates. In their model a choice between the migration and either proliferation or apoptosis depended on the local malignant cell density.

In this paper a deterministic reaction-diffusion model of invasive tumour growth is considered taking into account migration-proliferation dichotomy. A malignant cell can either proliferate or migrate. Transitions between these two stages (phenotypes) are supposed to depend on oxygen level in a threshold manner. High oxygen concentration allows cell proliferation, while concentration below a certain critical value induces cell migration. The mathematical model is investigated both analytically and numerically.

\section{The model}

We consider a tumour as a colony of living and dead cells surrounded by normal tissue. Living cells are also subdivided into two types: proliferating and migrating. A proliferating malignant cell divides with a constant rate but are immotile, a migrating cell can move in a random way but do not divide. Transitions between these two populations depend on oxygen (nutrient) concentration in a threshold manner. Concentration above the critical value causes cell proliferation, concentration below this value causes migration. If migrating malignant cells cannot reach region with appropriate oxygen level, they start to die. In the model, we take into account that though a malignant cell can divide under hypoxia, the oxygen shortage decreases rate of proliferation dramatically [11]. We consider a tumour growing in a normal tissue with rather poor capillary system, so oxygen diffuses from blood vessels located sufficiently away from the tumour. Both proliferating and migrating malignant cells consume oxygen, however, proliferating malignant cells consume nutrient much faster than migrating ones. Normal cells oxygen consumption has been neglected. Normal tissue surrounding the tumour is also supposed to hinder neither cancer cell motion nor proliferation. We restrict our analysis to a single spacial dimension case. Formally, the model is based on the following physical and biological assumptions:

- A tumour grows as a colony of proliferating, migrating, and dead cells with densities $n_{1}, n_{2}$, and $m$, respectively.

- Proliferating cells divide with a constant rate $B$ and in the case of low oxygen concentration 
$s$ pass into migration state with the rate $P_{1}(s)$. For sufficiently small oxygen concentration $P_{1}(s)$ should exceed $B$, else this transition will not occur.

- Migrating cells move randomly with coefficient $D_{n}$ and in the regions with high oxygen level can be again recruited into proliferation with the rate $P_{2}(s)$.

- Migrating malignant cells die with the constant rate $d$.

- Transitions between proliferating and migrating states and and vice versa depend on oxygen concentration in a threshold manner.

- Oxygen is considered to be a crucial nutrient for tumour growth. Its distribution is governed by diffusion $D_{s}$, while consumption is governed by living malignant cells both proliferating and migrating. We assume that cells consume oxygen with constant rate if its concentration is not too small.

Using these assumptions the governing equations can be written as follows:

$$
\begin{aligned}
\frac{\partial n_{1}}{\partial t} & =B n_{1}-P_{1}(s) n_{1}+P_{2}(s) n_{2} \\
\frac{\partial n_{2}}{\partial t} & =D_{n} \frac{\partial^{2} n_{2}}{\partial x^{2}}+P_{1}(s) n_{1}-P_{2}(s) n_{2}-d n_{2}, \\
\frac{\partial m}{\partial t} & =d n_{2} \\
\frac{\partial s}{\partial t} & =D_{s} \frac{\partial^{2} s}{\partial x^{2}}-q \frac{s}{s+s^{*}}\left(K n_{1}+n_{2}\right) .
\end{aligned}
$$

The transition rates $P_{1}(s)$ and $P_{2}(s)$ are step-like functions with a common critical oxygen level $S_{\text {crit }}$, width $1 / \varepsilon$ and amplitude $P$ :

$$
P_{1}(s)=P \frac{1-\tanh \left[\left(s-S_{\text {crit }}\right) \varepsilon\right]}{2}, \quad P_{2}(s)=P \frac{1-\tanh \left[\left(S_{\text {crit }}-s\right) \varepsilon\right]}{2} .
$$

So, oxygen concentrations which deviate from $S_{\text {crit }}$ over the values of the order of $1 / \varepsilon$ correspond to transition functions extreme values ( 0 or $P$ ).

In (2.1) the third equation decouples from the remaining system. The dead cells density profile determines the position of necrotic area inside the tumour. Therefore it does not affect the tumour spreading, which is governed only by the dynamics of proliferating, migrating malignant cells and nutrient concentration, and thus in our further analysis we omit this equation in the system (2.1).

Also we take into account that oxygen concentration does not fall well below the value $S_{\text {crit }}$ when all the cells die and oxygen consumption ceases. Thus, if we assume that $s^{*} \ll S_{\text {crit }}$ then the ratio $s /\left(s+s^{*}\right)$ in the last equation of (2.1) does not significantly deviate from unity and in our further analysis we omit it. 
At the avascular stage a tumour is supposed to have a spherically symmetric shape. However, if a tumour radius is much greater than the characteristic scale, for which cell density and nutrient concentration distributions change significantly, a planar geometry can be considered. By our assumption that the tumour grows in the tissue where oxygen predominantly diffuses from the blood vessels located far away from the tumour, the system (2.1) can be solved in an infinite region. Thus, we supplement equations (2.1) with the following boundary conditions:

$$
\left\{\begin{array}{l}
n_{1}=0, \\
n_{2}=0, \\
s_{x}=0,
\end{array} \quad x \rightarrow-\infty, \quad\left\{\begin{array}{l}
n_{1}=0, \\
n_{2}=0, \\
s=S_{\max },
\end{array} \quad x \rightarrow+\infty\right.\right.
$$

\section{Travelling wave solutions}

We seek the solution to (2.1) in the form of a wave travelling with a constant shape and speed $c$ (i.e. an autowave). Therefore, the governing equations (2.1) are reduced to a set of ordinary differential equations by introducing the travelling coordinate frame $\xi=x-c t$ :

$$
\begin{aligned}
c \frac{\partial n_{1}}{\partial \xi}+B n_{1}-P_{1}(s) n_{1}+P_{2}(s) n_{2} & =0 \\
D_{n} \frac{\partial^{2} n_{2}}{\partial \xi^{2}}+c \frac{\partial n_{2}}{\partial \xi}+P_{1}(s) n_{1}-P_{2}(s) n_{2}-d n_{2} & =0 \\
D_{s} \frac{\partial^{2} s}{\partial \xi^{2}}+c \frac{\partial s}{\partial \xi}-q\left(K n_{1}+n_{2}\right) & =0
\end{aligned}
$$

with the following boundary conditions:

$$
\left\{\begin{array}{l}
n_{1}=0, \\
n_{2}=0, \\
s=\sigma,
\end{array} \quad \xi \rightarrow-\infty, \quad\left\{\begin{array}{l}
n_{1}=0, \\
n_{2}=0, \\
s=1,
\end{array} \quad \xi \rightarrow+\infty .\right.\right.
$$

Here $\sigma$ corresponds to the limiting constant value of the substrate concentration for $\xi \rightarrow-\infty$. On the first step we consider the asymptotic behavior of the solutions to (3.1) for $\xi \rightarrow \pm \infty$. In order to do this we linearize equations (3.1) near the values (3.2) which are stationary points of (3.1) and obtain two sets of ODEs with constant coefficients

$$
\begin{aligned}
c \frac{\partial n_{1}^{-}}{\partial \xi}+B n_{1}^{-}-P_{1}(\sigma) n_{1}^{-} & =0, \\
D_{n} \frac{\partial^{2} n_{2}^{-}}{\partial \xi^{2}}+c \frac{\partial n_{2}^{-}}{\partial \xi}+P_{1}(\sigma) n_{1}^{-}-d n_{2}^{-} & =0 \\
D_{s} \frac{\partial^{2} s^{-}}{\partial \xi^{2}}+c \frac{\partial s^{-}}{\partial \xi}-q\left(K n_{1}^{-}+n_{2}^{-}\right) & =0
\end{aligned}
$$


and

$$
\begin{aligned}
& c \frac{\partial n_{1}^{+}}{\partial \xi}+B n_{1}^{+}+P n_{2}^{+}=0, \\
& D_{n} \frac{\partial^{2} n_{2}^{+}}{\partial \xi^{2}}+c \frac{\partial n_{2}^{+}}{\partial \xi}-P n_{2}^{+}-d n_{2}^{+}=0, \\
& D_{s} \frac{\partial^{2} s^{+}}{\partial \xi^{2}}+c \frac{\partial s^{+}}{\partial \xi}-q\left(K n_{1}^{+}+n_{2}^{+}\right)=0 .
\end{aligned}
$$

According to the linear differential calculus the solutions to these problems are sought in the form $\left(n_{1}, n_{2}, n_{2 \xi}, s, s_{\xi}\right)^{T} \sim \mathbf{k}^{ \pm} \exp \left(\mu^{ \pm} \xi\right)$ which reduces linear differential equations sets to eigenvalue problems for coefficients $\mu^{ \pm}$as eigenvalues and constant vectors $\mathbf{k}^{ \pm}$as eigenvectors. Eigenvalues $\mu^{ \pm}$indicate the rate of solutions exponential convergence (divergence) to the boundary values (3.2) as $\xi \rightarrow \pm \infty$ and superscripts ' + ' and '-' refer to the linearized problem on $+\infty$ and $-\infty$, respectively.

For the case $\xi \rightarrow+\infty$ it appears that the linearized set of ODEs (3.4) has three solutions with the rate of exponential convergence to the boundary conditions given by $\mu_{1}^{+}=-B / c, \mu_{2}^{+}=$ $-c / D_{s}$ and $\mu_{3}^{+}=\left(-c-\sqrt{c^{2}+4(P+d) D_{n}}\right) / 2 D_{n}$, one solution unbounded for $\xi \rightarrow+\infty$ with the rate of exponential divergence $\mu_{4}^{+}=\left(-c+\sqrt{c^{2}+4(P+d) D_{n}}\right) / 2 D_{n}$, and a single solution with a zero eigenvalue, $\mu_{5}^{+}=0$, of exponential growth. It can be shown that the stationary point $\left\{S 1: n_{1}=0, n_{2}=0, s=1\right\}$ of (3.1) is a saddle-node.

In the same manner, $\xi \rightarrow-\infty$ we derive the set of ODEs (3.3) linearized near the boundary conditions (3.2) for the case. Two solutions can be easily found: one with zero eigenvalue $\mu_{1}^{-}=$ 0 and the unbounded one corresponding to $\mu_{2}^{-}=-c / D_{s}$. Three others are roots of the cubic polynomial:

$$
c D_{n} \mu^{3}+\left(B D_{n}+c^{2}-P_{1}(\sigma)\right) \mu^{2}+c(B-d-P) \mu-B d-B P_{2}(\sigma)+d P_{1}(\sigma)=0 .
$$

A localized solution of equations (3.1), (3.2) exists only if at least one root of equation (3.5) is a real positive number. Further analytical treatment is possible only for the case $\left(S_{\text {crit }}-\sigma\right) \varepsilon \gg 1$. Then equation (3.5) is reduced to the following form:

$$
(c \mu+B-P)\left(D_{n} \mu^{2}+c \mu-d\right)=0 .
$$

Therefore, we get one more unbounded solution with $\mu_{3}^{-}=\left(-c-\sqrt{c^{2}+4 d D_{n}}\right) / 2 D_{n}$ and two solutions with exponential convergence coefficients to the boundary conditions $\mu_{4}^{-}=(-c+$ $\left.\sqrt{c^{2}+4 d D_{n}}\right) / 2 D_{n}$ and $\mu_{5}^{-}=(P-B) / c$. In this case stationary point $\left\{S 1: n_{1}=0, n_{2}=\right.$ $0, s=\sigma\}$ of (3.1) is also a saddle-node. It should be noted that such rough estimation does not impose any significant restriction (besides $c>0$ ) on the autowave speed. 


\section{Numerical simulations}

Equations (2.1) are solved numerically in a sufficiently large domain, so that the boundary conditions (2.3) imposed at the edge points of the space grid do not influence the solution. For our numerical algorithm we use the splitting method with respect to physical processes. Initially, we solve ODEs set which describes cell proliferation, transitions and death processes as well as nutrient consumption using the fourth order Runge-Kutta algorithm. As a next step, mass transfer equations for oxygen and cells are solved with the Crank-Nicolson method of the second order approximation in both space and time.

Any mathematical model of biological system depends crucially on parameter values choice. The parameters vary drastically depending on tumour type, localization, progression, etc. Parameters choice in the model is determined by the objective which is rather a qualitative characterization of an infiltrative tumour growth considering migration-proliferation dichotomy, than a quantitative description of any specific tumour. Therefore, the parameter values are taken in the experimentally observable range, and they are not related to a certain kind of cancer. The other important limitation on parameters is that their values are chosen in such a way that the tumour cell density remains substantially smaller, than the maximal possible value. This feature is typical for the infiltrative type tumour.

Equations (2.1) are already given in a non-dimensional form. In order to estimate the corresponding parameters we take characteristic scales of time and length: $T_{0}=10^{4} \mathrm{~s}$ and $L=$ $5 \cdot 10^{-2} \mathrm{~cm}$, respectively. The cell density is scaled on $n_{\max }=10^{7}$ cells $/ \mathrm{cm}^{3}$ (maximal cell density is the same for both proliferating and migrating cells), while the oxygen concentration in the tissue near blood vessels is supposed to be $S_{\max }=10^{-7} \mathrm{~mol} / \mathrm{cm}^{3}$. In dimensional values the cell proliferation rate corresponds to the cell division frequency of the order of one division per day or two. The diffusion coefficients for oxygen and cells are equal to $D_{s}=2.5 \cdot 10^{-5} \mathrm{~cm}^{2} / \mathrm{s}$ and $D_{n}=2.5 \cdot 10^{-9} \mathrm{~cm}^{2} / \mathrm{s}$, respectively. Thus, we obtain the following non-dimensional parameters of the problem: $D_{n}=0.01, D_{s}=100, B=0.05, P=0.1, d=0.1, S_{\text {crit }}=0.3, \varepsilon=100, q=0.2$, $k=10$, which will be referred to as a standard parameter set.

We suppose that an oxygen concentration is initially maximum everywhere: $s=1$ and there are no malignant cells of any type: $n_{1}=n_{2}=m=0$ except a low number of proliferating cells placed near the left border of the region under consideration. During initial growth period malignant cells only divide due to high oxygen concentration in the tissue. When in the primary tumour site a nutrient concentration drops below the critical value $S_{\text {crit }}$, migrating malignant cells appears. There is a single source of oxygen on the right border, thus if migrating cells move towards the left border they get into hypoxia zone and sooner or later die. Thereby, tumour spreads (grows) to the nutrient source on the right border.

While the tumour spreading speed remains in fact constant from the beginning of growth, tumour cell density decreases with time (see Fig. 1). It should be also noted, that active malignant cells are located only in a thin layer on the tumour border. It happens due to oxygen depletion inside the tumour below the value $S_{\text {crit }}$ necessary for proliferation. Numerical simulation shows, that oxygen concentration on the left border $\sigma$ is only slightly less than this critical value $\left(S_{\text {crit }}-\sigma\right) \varepsilon \sim$ 1 , so oxygen consumption inside the tumour is negligible. Tumour interior consists of necrotizing 


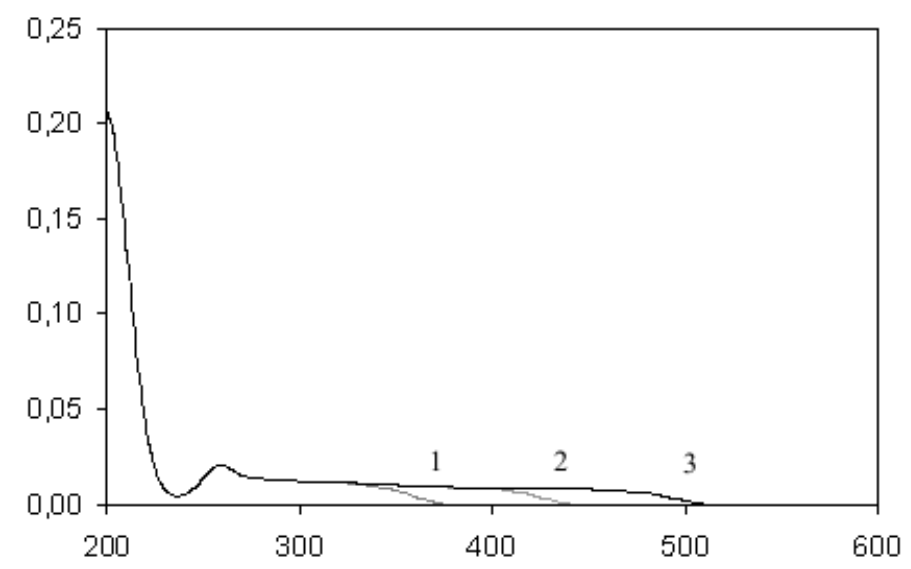

Figure 1: Overall tumour density $n_{1}+n_{2}+m$ for the standard parameter set at various moments of growth: (1) $T=200$, (2) $T=400$, (3) $T=600$.

cells and a rather dense necrotic core is observed near the primary site $(x=200)$ of malignant cell inoculation.

In the course of time proliferating and migrating cells profiles become constant (autowave distribution) in the frame moving with the tumour growth speed. These profiles for the standard parameter set are demonstrated in Fig. 2. It should be noted, that both proliferating and migrating cell distributions are coupled. Perhaps, such a behavior occurs due to critical oxygen concentrations for the transitions from proliferation to migration and backward being taken the same.

The other important problem solved via numerical computations is a dependence of the tumour growth speed $c$ on parameter values. Alteration of parameters $D_{s}, q$, and $k$ of the fourth equation from the set (2.1) does not affect the tumour growth velocity $c$ anyway. These parameters influence only malignant cell spatial distributions, but not the tumour spreading rate. Numerical study also demonstrates that tumour growth speed, like in the Kolmogorov-Petrovskii-Piskunov (KPP) or Fisher equations, is strictly proportional to the square root of cell migration coefficient $D_{n}(c \sim$ $\left.D_{n}^{1 / 2}\right)$. The dependencies of $c$ on cell division rate $B$ and cell death rate $d$ are not so trivial. They are shown in Fig. 3. (In our simulations a variation of the parameter $B$ was accompanied by simultaneous alteration of the transition function amplitudes $P$ so that $P=2 B$.) The reason of these dependencies is still unclear.

From our point of view, a very important relationship is observed between the tumour growth speed $c$ and the ratio of oxygen concentration in tissue $S_{\max }$ to the level necessary for proliferation $S_{\text {crit }}$. This dependence is shown in Fig. 4. We remind that oxygen concentration in the tissue near blood vessels $S_{\max }$ is a scaling parameter equal to unity. Thus, one can see that, when the oxygen concentration in tissue is far from the critical value required for cell proliferation, the tumour growth speed is practically constant, while for the oxygen concentrations near this critical value the tumour growth speed decreases dramatically. 


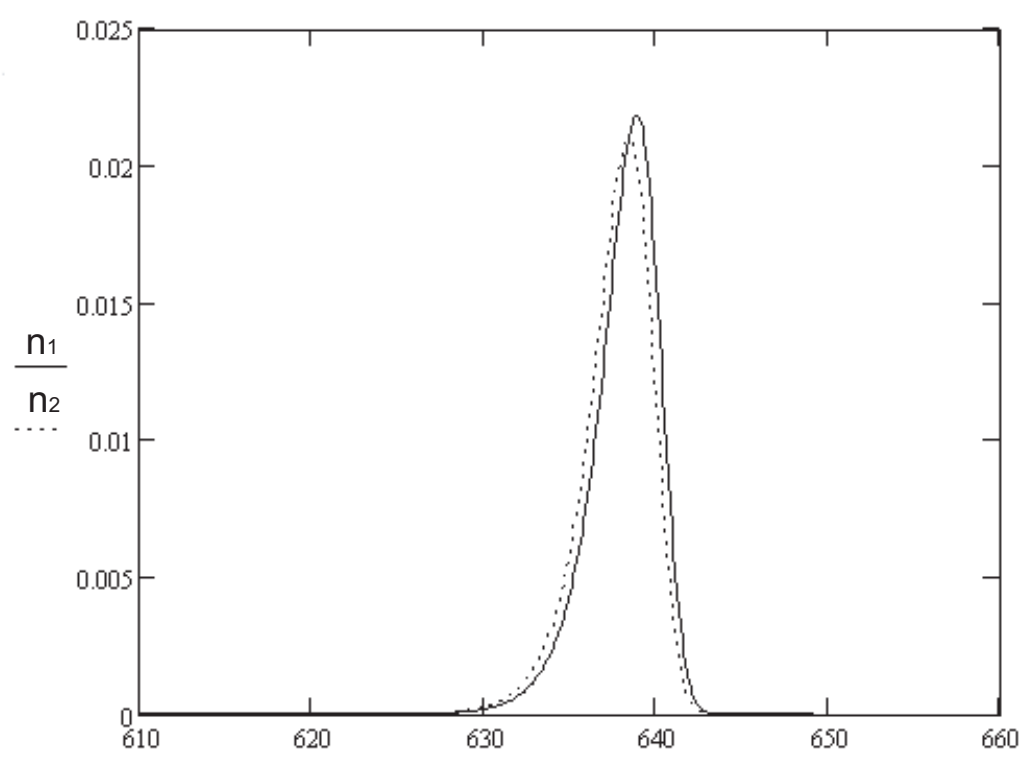

Figure 2: Proliferating $n_{1}$ (solid line) and migrating $n_{2}$ (dashed line) cell densities profiles as a function of coordinate $x$ for the standard parameter set at the moment $T=700$.
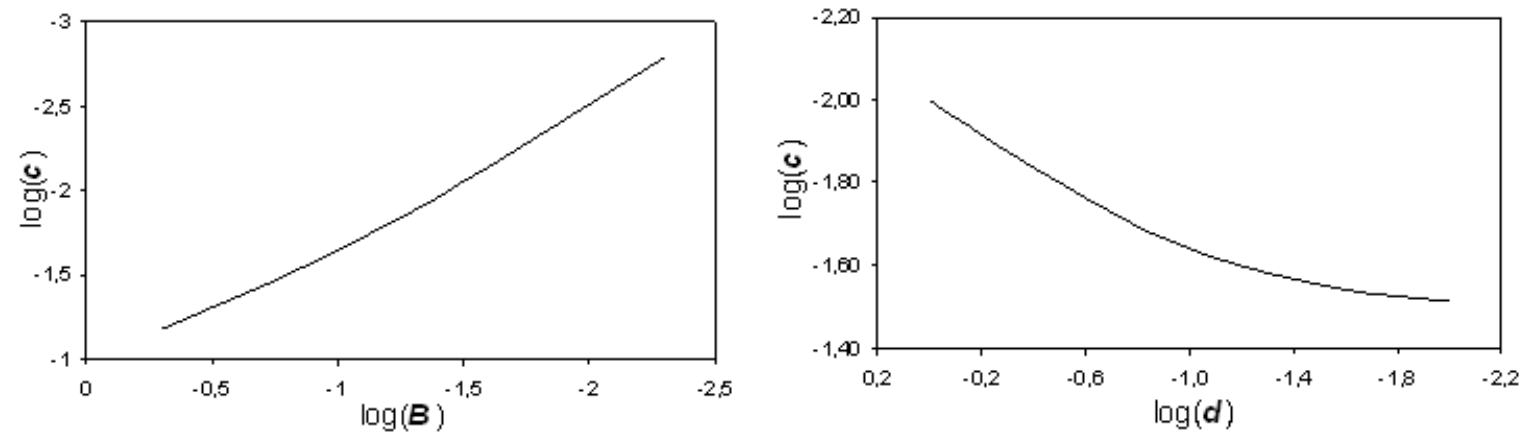

Figure 3: Dependencies of the tumour growth rate $c$ on proliferation rate $B$ (left) and death rate $d$ (right) in logarithmic scale. 


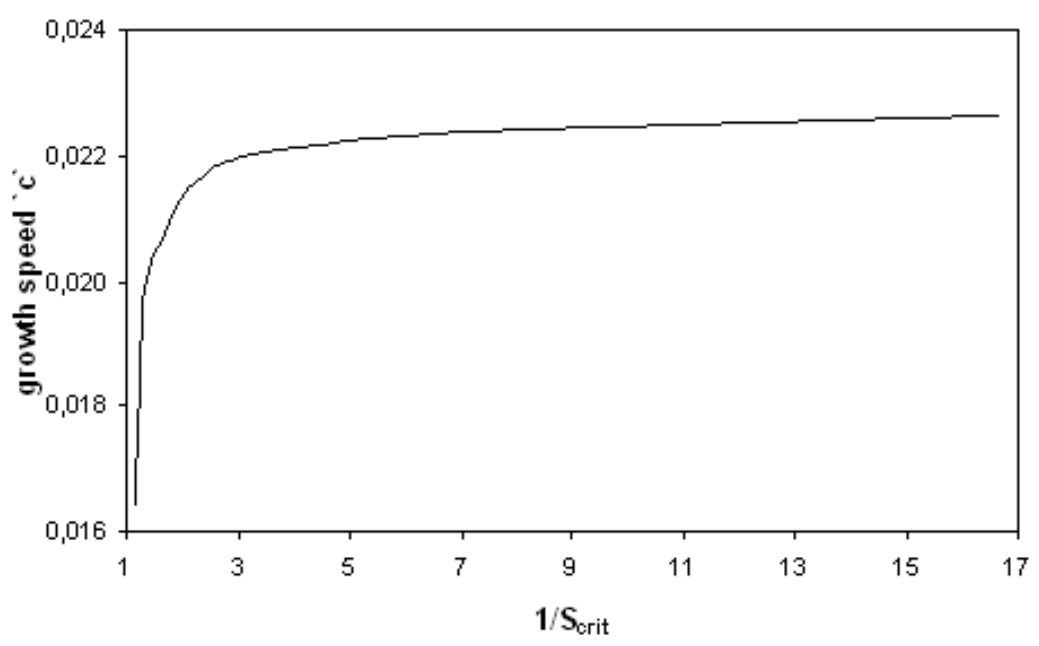

Figure 4: Dependence of tumour growth rate $c$ on inverse critical oxygen concentration $1 / S_{\text {crit }}$ for the standard set of parameters.

\section{Discussion}

In this paper a mathematical model of infiltrative tumour growth with account of migrationproliferation dichotomy is studied. The model adequately describes a constant rate of the tumour linear size growth at the initial stage of neoplasm development and the necrotic region formation in the tumour interior, observed in the experiments.

The evolution of malignant cell density profiles is investigated. It is shown that the initially localized distribution asymptotically approaches the automodel solution traveling with a constant speed. As long as this speed is proportional to the square root of cell migration coefficient, it resembles the autowave solutions behavior of the Fisher equation. However, in contrast to the Fisher model the velocity dependence on the malignant cell proliferation rate is more similar to the pushed regime as formulated in [17]. We suppose, that in fact the autowave speed selection occurs on the left asymptotic when the discriminant of the cubic polynomial equation (3.5) becomes negative. This issue is the subject of our future study.

Numerical simulations of the model demonstrate that the tumour growth rate depends on the tissue oxygen level in a threshold manner. If oxygen level is far from the critical value required for cell proliferation, tumour growth speed is insensitive to oxygen decrease, while for oxygen concentrations near this critical value the tumour growth speed decreases dramatically. This result may be considered as additional evidence in favour of tumour antiangiogenic therapy. The aim of this therapy is to create a hypoxic region around the tumour which, according to our model, should slow down the tumour growth and even can completely stop it.

On the present stage, we limited our study to the case of equal threshold oxygen concentrations for the transitions from proliferation to migration and backwards. However, it is clear, that the model should be studied for all parameter values of transition functions. It is supposed to be a subject of our further investigations. 


\section{Acknowledgements}

The authors acknowledge the financial support from Dynasty Foundation and RFBR: grants No. 08-01-00131, 10-01-00289, and 08-02-00806.

\section{References}

[1] A.C. Burton. Rate of growth of solid tumours as a problem of diffusion. Growth, 30 (1966), 157-176.

[2] H. Bueno, G. Ercole, A. Zumpano. Asymptotic behaviour of quasi-stationary solutions of a nonlinear problem modelling the growth of tumours. Nonlinearity, 18 (2005), 1629-1642.

[3] H.M. Byrne, M.A.J. Chaplain. Growth of necrotic tumours in the presence and absence of inhibitors. Math. Biosci., 135 (1996), 187-216.

[4] S.K. Chintala, J.R. Rao. Invasion of human glioma: role of extracellular matrix proteins. Frontiers in Bioscience, 1 (1996), 324-339.

[5] S. Fedotov, V. Mendez. Continuous-time random walks and traveling fronts. Phys. Rev. E, 66 (2002), 030102.

[6] S. Fedotov, A. Iomin. Migration and Proliferation Dichotomy in Tumor-Cell Invasion. Phys. Rev. Lett., 98 (2007), 118101.

[7] A. Giese, R. Bjerkvig, M.E. Berens, M. Westphal. Cost of migration: invasion of malignant gliomas and implications for treatment. J. Clinical Oncology, 21 (2003), 1624-1636.

[8] H.P. Greenspan. On the growth and stability of cell cultures and solid tumors. J. Theor. Biol., 56 (1976), 229-242.

[9] A. Gusev, A. Polezhaev. Modelling of a cell population evolution for the case existence of maximal possible total cell density. Kratkie soobscheniya po fizike FIAN, 11-12 (1997), 8590.

[10] D. Hanahan, R.A. Weinberg. The hallmarks of cancer. Cell, 100 (2000), 57-70.

[11] A.L. Harris. Hypoxia - a key regulatory factor in tumour growth. Nat. Rev. Cancer, 2 (2002), $38-47$.

[12] H. Hatzikirou, D. Basanta, M. Simon, K. Schaller, A. Deutsch. 'Go or Grow': the key to the emergence of invasion in tumour progression? Math. Med. Biol., 7 (2010), 1-17.

[13] A. Iomin. Toy model of fractional transport of cancer cells due to self-entrapping. Phys. Rev. E, 73 (2006), 061918. 
[14] A.V. Kolobov, A.A. Polezhaev, G.I. Solyanyk. Stability of tumour shape in pre-angiogenic stage of growth depends on the migration capacity of cancer cells. Mathematical Modelling \& Computing in Biology and Medicine (Ed. V. Capasso), 2003, 603-609.

[15] A.K. Laird. Dynamics of tumor growth. Br. J. Cancer, 18 (1964), 490-502.

[16] J.S. Lowengrub, H.B. Frieboes, F. Jin, Y-L. Chuang, X. Li, P. Macklin, S.M. Wise, V. Cristini. Nonlinear modelling of cancer: bridging the gap between cells and tumours. Nonlinearity, 23 (2010), 1-91.

[17] W. van Saarloos. Front propagation into unstable states. Physics Reports, 386 (2003), 29222.

[18] J.A. Sherratt, M.A.J. Chaplain. A new mathematical model for avascular tumour growth. J. Math. Biol., 43 (2001), 291-312.

[19] K.R. Swansona, C. Bridge, J.D. Murray, E.C. Alvord Jr. Virtual and real brain tumors: using mathematical modeling to quantify glioma growth and invasion. J. Neurolog. Sci., 216 (2003), 1-10.

[20] Y. Tao, M. Chen. An elliptic-hyperbolic free boundary problem modelling cancer therapy. Nonlinearity, 19 (2006), 419-440.

[21] R.H. Thomlinson, L.H. Gray. The histological structure of some human lung cancers and the possible implications for radiotherapy. Br. J. Cancer, 9 (1955), 539-549.

[22] J.P. Ward, J.R. King. Mathematical modelling of avascular-tumour growth. IMA J. Math. Appl. Med. Biol., 14 (1997), 39-69. 